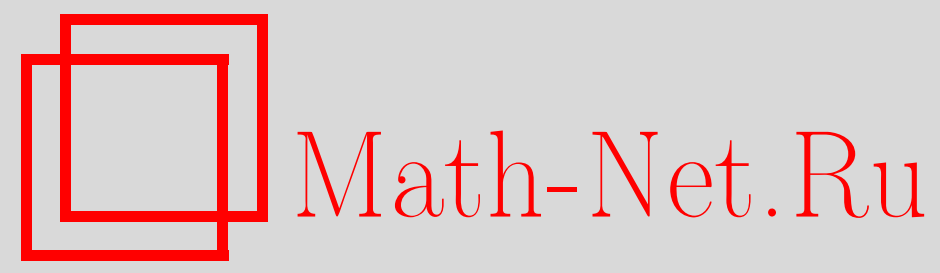

О. К. Закусило, Критерий существования локального времени в нуле для одного класса процессов хранения, Теория вероятн. и ее примен., 1996, том 41, выпуск 1, 200-203

DOI: https://doi.org/10.4213/tvp2794

Использование Общероссийского математического портала Math-Net.Ru подразумевает, что вы прочитали и согласны с пользовательским соглашением http://www . mathnet.ru/rus/agreement

Параметры загрузки:

IP: 3.93 .64 .190

26 апреля 2023 г., $14: 15: 26$

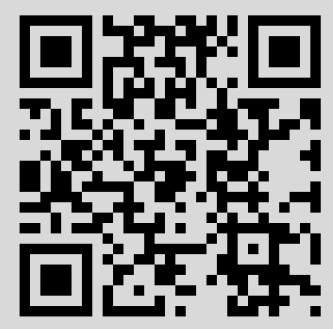


(c) $1996 \mathrm{r}$.

ЗАКУСКЛО О. К.*

\section{КРИТЕРИЙ СУЩЕСТВОВАНИЯ ЛОКАЛЬНОГО ВРЕМЕНИ В НУЛЕ ДЛЯ ОДНОГО КЛАССА ПРОЦЕССОВ ХРАНЕНИЯ}

В данной заметке завершены исследования, проведенные в работах [1]-[3] при изучении условий сушествования локального времени в нуле $L(t)$ для процесса хранения запасов $x(t)$, являющегося решением уравнения (1) с характеристиками (2). Кроме того, указана структура множества нулей $E$.

Ключевые слова и фразы: процесс хранения запасов, локальное время, входной процесс, мера Леви.

Пусть $x(t) \geqslant 0$ есть процесс хранения запасов, являющийся решением уравнения

$$
d x(t)=-r(x(t)) d t+d z(t)
$$

в котором $z(t)$ - неубываюший непрерывный справа однородный процесс с независимыми приращениями и с преобразованием Лапласа

$$
\mathbf{E} \exp \{-s z(t)\}=\exp \left\{-t \int_{0}^{\infty}\left(1-e^{-s u}\right) \nu(d u)\right\}
$$

a $r(x)$ - неубывающая и непрерывная на $(0, \infty)$ функция, $r(x)>0$ при $x>0, r(0)=0$.

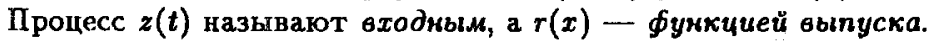

В настоящей заметке мы завершаем исследования, проведенные в работах [1]-[3] при изучении условий существования локального времени в нуле $L(t)$ для процессов (1) с характеристиками

$$
\nu(d x)=A x^{-1-\alpha} d x, \quad 0<\alpha<1, \quad r(x)=x^{\beta}, \quad \beta>0 .
$$

Кроме того, мы указываем структуру множества нулей $E=\{t>0: x(t)=0\}$ при различных значениях $\alpha, \beta$ и $A$.

Наиболее полное утверждение о сушествовании $L(t)$ для процессов (1) с характеристиками (2) содержится в работе [3]:

а) если $\alpha+\beta<1$, то $L(t)$ существует;

б) если $\alpha+\beta>1$, то $L(t)$ не существует;

в) если $\alpha+\beta=1$, то $L(t)$ существует при $A<A_{0}=A_{0}(\alpha)$ и не сушествует при $A>A_{0} ;$ при этом $A_{0} \in(0, \infty)$.

При исследовании общих процессов (1) с бесконечными мерами Леви $\nu$ Цинлар [4] показал, что при $x(0)=0$ почти наверное справедливо одно из трех утверждений:

г) замыкание $E$ не содержит интервалов, $E$ не содержит изолированных точек, лебегова мера $E$ положительна, $L(t)$ лишь множителем отличается от времени пребывания в нуле;

д) $E$ имеет ту же структуру, что и в г), но лебегова мера $E$ равна нулю, а $L(t)$ не совпадает (с точностью до множителя) со временем пребывания в нуле;

е) $E=\varnothing, L(t)$ не существует.

Мы найдем значение $A_{0}$, покажем, что при $A=A_{0}$ локальное время в нуле $L(t)$ не сушествует и установим, что все гипотетические возможности г)-е) могут быть реализованы процессами хранения с характеристиками (2).

*Ккевский национальный университет им. Т. Г. Шевченко, ул. Владимирская, 64, 252017 Киев, Украина. 
Введем необходимые обозначения:

$$
\tau(y)=\inf \{t>0: x(t)=y\}, \quad \varphi(s, x, y)=\mathbf{E}_{x} e^{-s \tau(y)},
$$

$\mathbf{E}_{\boldsymbol{x}}$ - символ математического ожидания случайной величины, связанной с процессом $x(t)$, для которого $x(0)=x$.

Теорема 1. Для прочесса храяения (1) со степекяой фуякцией выпуска $r(x)=$ $x^{1-\alpha}$ и со строго устойчивым өходньм прочессом с мерой $Л е в и ~ \nu(d u)=A u^{-1-\alpha} d u$ локальное өремя в куле существует при $A<A_{0}=\alpha \sin (\alpha \pi) / \pi$ и не существует при $A \geqslant A_{0}$.

Д ок а за т е льст в о. І. Пусть $A \geqslant A_{0}$. В этом случае можно почти дословно повторить рассуждения статьи [2], проведенные для процессов хранения с

$$
\nu(d u)=\frac{\alpha}{\Gamma(1-\alpha)} u^{-1-\alpha} d u .
$$

Введем $F(x)=1-\varphi(s, x, 0)$. Из результатов работы [4] следует, что $F(x)-$ непрерывная справа функция распределения, удовлетворяющая на интервале $(0, \infty)$ уравнению

$$
F^{\prime}(x)-s x^{-1+\alpha}(1-F(x))=\frac{A}{\alpha} x^{-1+\alpha} \int_{x}^{\infty}(u-x)^{-\alpha} d F(u)
$$

Интегрируя его по интервалу $(0, \infty)$, меняя порядок интегрирования в правой части и проводя замену $\boldsymbol{x}=\boldsymbol{u z}$, получаем

$$
\begin{aligned}
1-F(0)-s \int_{0}^{\infty} x^{-1+\alpha}(1-F(x)) d x & =\frac{A}{\alpha} \int_{0}^{\infty} \int_{0}^{1} z^{-1+\alpha}(1-z)^{-\alpha} d z d F(u) \\
& =\frac{C A}{\alpha}(1-F(0)),
\end{aligned}
$$

где

$$
C=\int_{0}^{1} z^{-1+\alpha}(1-z)^{-\alpha} d z=\frac{\pi}{\sin \pi \alpha}
$$

Значит,

$$
s \int_{0}^{\infty} x^{-1+\alpha}(1-F(x)) d x=(1-F(0))\left(1-\frac{C A}{\alpha}\right) .
$$

Простой анализ знаков частей последнего равенства показывает, что при $A \geqslant A_{0}$ оно возможно лишь в случае $F(0)=1$. Но тогда $F(x)=1$ при всех $x \geqslant 0, \varphi(s, x, 0)=0$ при $x>0, \mathbf{P}_{x}\{\tau(0)=\infty\}=1$ при $x>0$.

Последнее равенство с учетом утверждения (2.31) работы [4] позволяет утверждать, что в рассматриваемой ситуации $L(t)$ не существует.

II. Пусть $A<A_{0}$. Для проверки существования $L(t)$ в силу утверждения $(2.31)$ работы [4] нужно установить, что $\varphi(s, x, 0)>0$ для некоторых $s>0, x>0$. Для этого, в свою очередь, достаточно убедиться в существовании функции $h(x)$, удовлетворяющей условиям

$$
\infty>h(x)>0, \quad h(x) \geqslant s+\int_{0}^{\infty}(1-H(x, x+v)) \nu(d v)
$$

где

$$
H(y, x)=\exp \left\{-\int_{y}^{x} h(u) \frac{d u}{r(u)}\right\}>0 \quad \text { при } \quad x>y .
$$

(Как показано в работе [3], $\varphi(s, x, 0) \geqslant H(0, x)$, что и даст требуемое условие $\varphi(s$, $x, 0)>0$.) 
Покажем, что функция $h(x)=1+x^{-\varepsilon}$ удовлетворяет условиям (3) при

$$
\frac{\alpha}{2} \leqslant \varepsilon<\alpha, \quad \frac{A}{\alpha} B(1-\alpha, \varepsilon)<1, \quad 0<s \leqslant 1-\frac{A}{\alpha} B(1-\alpha, \varepsilon),
$$

где $B(a, b)=\int_{0}^{1} x^{a-1}(1-x)^{b-1} d x$ есть бета-функция.

С учетом условия (2) имеем:

$$
\begin{aligned}
s & +\int_{0}^{\infty}(1-H(x, x+v)) \nu(d v)=s+\int_{0}^{\infty} \frac{H(0, x)-H(0, x+v)}{H(0, x)} \nu(d v) \\
& =s+\frac{A}{H(0, x)} \int_{0}^{\infty} \int_{x}^{x+v} H_{z}^{\prime}(0, z) d z v^{-1-\alpha} d v \\
& =s+\frac{A}{\alpha H(0, x)} \int_{x}^{\infty}(z-x)^{-\alpha} H_{z}^{\prime}(0, z) d z \\
& =s+\frac{A}{\alpha} \int_{x}^{\infty}(z-x)^{-\alpha} \exp \left\{-\int_{x}^{z}\left(1+u^{-\varepsilon}\right) u^{\alpha-1} d u\right\}\left(1+z^{-\varepsilon}\right) z^{\alpha-1} d z \\
& =s+\frac{A}{\alpha} \int_{1}^{\infty}(v-1)^{-\alpha} \exp \left\{-\int_{x}^{v x}\left(1+u^{-\varepsilon}\right) u^{\alpha-1} d u\right\}\left(1+(v x)^{-\varepsilon}\right) v^{\alpha-1} d v \\
& =s+\frac{A}{\alpha}\left(1+x^{-\varepsilon}\right) \int_{1}^{\infty}(v-1)^{-\alpha} v^{\alpha-1-\varepsilon} Q(x, v) d v
\end{aligned}
$$

где

$$
Q(x, v)=\exp \left\{-\int_{x}^{v x}\left(1+u^{-\varepsilon}\right) u^{\alpha-1} d u\right\} \frac{1+(v x)^{-\varepsilon}}{1+x^{-\varepsilon}} v^{\varepsilon} .
$$

Если $0<\alpha / 2 \leqslant \varepsilon<\alpha<1$ и $v \geqslant 1$, то

$$
\begin{aligned}
\ln Q(x, v) & =-\int_{x}^{v x}\left[\left(1+u^{-\varepsilon}\right) u^{\alpha-1}-\frac{\varepsilon u^{\varepsilon-1}}{u^{\varepsilon}+1}\right] d u \\
& =-\int_{x}^{v x} \frac{2+u^{\varepsilon}+u^{-\varepsilon}-\varepsilon u^{\varepsilon-\alpha}}{u^{\varepsilon}+1} u^{\alpha-1} d u \leqslant 0
\end{aligned}
$$

и $Q(x, v) \leqslant 1$.

Поэтому из равенства (5) с учетом условий (4) следует условие (3):

$$
\begin{aligned}
s+ & \int_{0}^{\infty}(1-H(x, x+v)) \nu(d v) \leqslant s+\frac{A}{\alpha}\left(1+x^{-\varepsilon}\right) \int_{1}^{\infty}(v-1)^{-\alpha} v^{\alpha-1-\varepsilon} d v \\
& =s+\frac{A}{\alpha}\left(1+x^{-\varepsilon}\right) \int_{0}^{1}(1-z)^{-\alpha} z^{e-1} d z=s+\frac{A}{\alpha} B(\varepsilon, 1-\alpha)\left(1+x^{-\varepsilon}\right) \\
& \leqslant 1+x^{-e}=h(x) .
\end{aligned}
$$

В свою очередь, ввиду непрерывности бета-функции,

$$
B(1-\alpha, \varepsilon) \underset{\varepsilon \rightarrow \alpha}{\longrightarrow} B(1-\alpha, \alpha)=\frac{\pi}{\sin \alpha \pi}
$$

и условия (4) можно удовлетворить, если $A<A_{0}=\alpha \sin (\pi \alpha) / \pi$.

Этого достаточно для завершения доказательства.

Перейдем теперь к выяснению структуры множества нулей $E$ и локального времени в нуле $L(t)$.

Теорема 2. Eсли прочесс хракения имеет характеристики (2), то при $\alpha+\beta<$ 1 множество $E$ и локальное время $L(t)$ имеет структуру, указакную в пункте $r$ ), а при $\alpha+\beta=1$ и $A<A_{0}-$ структуру, указанкую в пункте д). 
Д ох а за те ль с тв о. Зафиксируем $s>0$ и обозначим $\varphi(x)=\varphi(s, x, 0)$. Цинлар [4] показал, что ситуация, указанная в пункте г), наблюдается при условиях $\nu(0, \infty)=\infty$ и $\int_{0}^{\infty}(1-\varphi(x)) \nu(d x)<\infty$. Если же $\nu(0, \infty)=\infty$ и $\int_{0}^{\infty}(1-\varphi(x)) \nu(d x)=$ $\infty$, то $E$ и $L(t)$ обладают свойствами, указанными в пункте д).

Пусть $\alpha+\beta<1$. Из лемм 3 и 4 работы [3] вытекает, что для достаточно больших значений $K$ функция $h(x)=K$ удовлетворяет неравенству (3) и

$$
\varphi(x) \geqslant \exp \left\{-\frac{K}{1-\beta} x^{1-\beta}\right\}
$$

Поэтому

$$
\int_{0}^{\infty}(1-\varphi(x)) \nu(d x) \leqslant A \int_{0}^{\infty}\left(1-\exp \left\{-\frac{K}{1-\beta} x^{1-\beta}\right\} x^{-1-\alpha} d x<\right) \infty
$$

Пусть теперь $\alpha+\beta=1, A<A_{0}$. Теорема 1 утверждает, что в этом случае $L(t)$ существует и, следовательно, $E \neq \varnothing$. С учетом результатов работы [4] отсюда вытекает, что $\varphi(0)=1, \varphi(x)>0$ при $x>0$. Это, в свою очередь, обеспечивает справедливость оценки, указанной в работе [3] для функции $g(x)=-(1 / r(x)) d \ln \varphi(x) / d x$ :

$$
g(x)>C x^{-A(1-\varepsilon) /(\alpha \varepsilon)}
$$

при достаточно малых $C, x$ и $\varepsilon$.

Далее, лемма 2 работы [3] утверждает, что

$$
g(x)=s+\int_{0}^{\infty}(1-\varphi(s, x+v, x)) \nu(d v)
$$

В этой же работе показано, что в наших условиях $\varphi(s, x+v, x)$ не убывает по $x$. Поэтому из непрерывности $\varphi(s, x+v, x)$ по $x$ при $x \in[0, \infty)$, равенства (7), теоремы о монотонной сходимости и оценки (6) следует:

$$
\int_{0}^{\infty}(1-\varphi(v)) \nu(d v)=\lim _{x \downarrow 0} \int_{0}^{\infty}(1-\varphi(s, x+v, x)) \nu(d x)=\lim _{x \downarrow 0}(g(x)-s)=\infty
$$

Вместе с равенством $\nu(0, \infty)=\infty$ это обеспечивает выполнение условий Цинлара, при которых справедливо утверждение пункта д).

\section{СПИСОК ЛИТЕРАТУРЫ}

1. Brockwell P. J., Chung K. L. Emptines times of a dam with stable input and a general release rule. - J. Appl. Probab., 1975, v. 12, № 1, p. 212-217.

2. Brockwell $P$. J. Stationary distributions for dams with additive input and contentdependent release rule. - Adv. Appl. Probab., 1977, v. 9, № 3, p. 645-663.

3. Закусило $О$. $K$. Еще раз о локальном поведении процессов хранения с устойчивым входом. - Теория вероятн. и ее примен., 1990, т. 35, в. 2, с. 349-352.

4. Cinlar E. A local time for a storage process. - Ann. Probab., 1975, v. 3, № 6, p. 930-951. 